Homology, Homotopy and Applications, vol.11(1), 2009, pp.171-184

\title{
A HOMOTOPICAL ALGEBRA OF GRAPHS RELATED TO ZETA SERIES
}

\author{
TERRENCE BISSON AND ARISTIDE TSEMO
}

(communicated by J. Daniel Christensen)

\begin{abstract}
The purpose of this paper is to develop a homotopical algebra for graphs, relevant to the zeta series and the spectra of finite graphs. More precisely, we define a Quillen model structure in a category of graphs (directed and possibly infinite, with loops and multiple arcs allowed). The weak equivalences for this model structure are the Acyclics (graph morphisms which preserve cycles). The cofibrations and fibrations for the model are determined from the class of Whiskerings (graph morphisms produced by grafting trees). Our model structure seems to fit well with the importance of acyclic directed graphs in many applications.
\end{abstract}

\section{Introduction}

In this paper we develop a notion of homotopy within graphs, and demonstrate its relevance to the study of the zeta series and the spectrum of a finite graph. We will work throughout with a particular category of graphs, described in Section 2 below. Our graphs will be directed and possibly infinite, with loops and multiple arcs allowed.

Let us explain what we mean by homotopy here.

We are not concerned with the geometric realization of graphs as one-dimensional topological spaces. Since one-dimensional CW complexes are homotopic to disjoint unions of joins of circles, the usual invariants from algebraic topology cannot see much of the structure of a graph in this way. In any case, directed graphs are definitely not just part of topology (they are perhaps more related to new areas of directed topology, as in Fajstrup and Rosický [5]).

Homotopy originally referred to topological deformation of structure. But Quillen's remarkable notes on homotopical algebra [14] gave abstract axioms for working with concepts of homotopy in rather general categories. When these axioms are satisfied in a category, we say that we have given a "model structure" there. Quillen's axioms have led to new insights and developments in settings such as chain complexes and homological algebra, simplicial sets, topos theory, and small categories (including

Received September 21, 2008, revised January 29, 2009; published on May 19, 2009.

2000 Mathematics Subject Classification: 05C20, 18G55, 55U35.

Key words and phrases: category of directed graphs, topos, Quillen model structure, weak factorization system, cycles, zeta function.

This article is available at http://intlpress.com/HHA/v11/n1/a8

Copyright (c) 2009, International Press. Permission to copy for private use granted. 
monoids, groups, groupoids, and posets). References include Cisinski [1], Dwyer and Spalinski [3], Hovey [6], Joyal and Tierney [7], Thomason [17], and many others. Also, recent proofs of the Bloch-Kato and Milnor conjectures are based upon development of a homotopical algebra for schemes; see Morel and Voevodsky [13].

A central part of giving a model structure in a category is the specification of which morphisms in the category are to be called "weak equivalences". In most applications, the weak equivalences are defined to be those morphisms which preserve some interesting invariant, such as homotopy type for topology, homology for chain complexes, geometric realization for simplicial sets, and nerve or topos of presheafs for small categories.

In our model structure we use the cycle structure of directed graphs to determine our weak equivalences. More precisely, we take as our weak equivalences the "acyclic" graph morphisms, which neither create nor destroy cycles. We hope that our model structure fits well with the role that acyclic directed graphs play in applications such as computer algorithms, analysis of the internet, random walks and Markov chains, and representations of quivers.

In Section 2 we set up our category Gph of graphs. In Section 3 we give background on weak factorization systems in general, and establish an example with classes of graph morphisms which we call Whiskerings and Surjectings. In Section 4 we give axioms for model structures in general, and define our model structure on Gph. In Section 5 we associate to each finite directed graph $X$ a zeta series $Z_{X}(u)$. We show that if $f: X \rightarrow Y$ is an acyclic graph morphism, then $Z_{X}(u)=Z_{Y}(u)$ and the eigenvalues of the adjacency matrices of $X$ and $Y$ agree "up to zero eigenvalues". The paper ends with an appendix on the history of zeta series.

\section{Acknowledgements}

The first author would like to thank André Joyal and Jiří Rosický for encouragement, York University for hospitality during fall 2005, and his students in the "Geometry and Physics on Graphs" Research Experience for Undergraduates at Canisius College. We thank the Fields Institute for work space, and the referee for comments and suggestions.

\section{A category of graphs}

Let us establish precisely the objects and morphisms for our category Gph of graphs. For our purposes, a graph is a data structure $X=\left(X_{0}, X_{1}, s, t\right)$ with a set $X_{0}$ of nodes, a set $X_{1}$ of arcs, and a pair of functions $s, t: X_{1} \rightarrow X_{0}$ which specify the source and target nodes of each arc. We may say that $a \in X_{1}$ is an arc from node $s(a)$ to node $t(a)$. A graph morphism $f: X \rightarrow Y$ is a pair of functions $f_{1}: X_{1} \rightarrow Y_{1}$ and $f_{0}: X_{0} \rightarrow Y_{0}$ such that $s \circ f_{1}=f_{0} \circ s$ and $t \circ f_{1}=f_{0} \circ t$.

Let $\mathbf{D}$ denote the graph with one node and no arcs. Let $\mathbf{A}$ denote the graph with one arc and two nodes (its source and target). Then the set of nodes of a graph $X$ can be identified with the set of graph morphisms from $\mathbf{D}$ to $X$, and the set of arcs of $X$ can be identified with the set of graph morphisms from $\mathbf{A}$ to $X$. 
There is a stimulating discussion in Lawvere [10] of Gph as the category of presheafs on the small category with objects 0 and 1 and two non-identity morphisms from 0 to 1 . It follows that Gph is a topos, and thus a category with many nice geometric and algebraic and logical properties; see Mac Lane and Moerdijk [12], for instance. We just call attention to a few aspects here.

The category Gph has all products, and all coproducts (sums); it also has pull-backs (fiber products) and pushouts. Also, products distribute over coproducts, etc. As in any presheaf category, these categorical constructions are performed "elementwise", where a graph has two types of elements, the nodes and the arcs. For instance, the empty product (terminal object) 1 is the graph with one node and one arc (which is a loop), and the empty coproduct (initial object) 0 is the graph with no nodes and no arcs.

The category Gph has geometric aspects; for instance, it is a cartesian closed category like a good category of "spaces". The category Gph also has logical aspects; for instance, there is a graph $\Omega$ which acts as generalized truth-values for graphs, in that graph morphisms $X \rightarrow \Omega$ classify sub-graphs of $X$ (see Session 32 in Lawvere and Schanuel [11]).

\section{Two classes of graph morphisms, and a weak factorization system}

The path graph $\mathbf{P}_{n}$ has nodes $\{i: 0 \leqslant i \leqslant n\}$ and $\operatorname{arcs}\{(i-1, i): 1 \leqslant i \leqslant n\}$, with $s((i-1, i))=i-1$ and $t((i-1, i))=i$. Note that $\mathbf{P}_{0}=\mathbf{D}$ and $\mathbf{P}_{1}=\mathbf{A}$. A path in a graph $X$ is just a graph morphism $\alpha: \mathbf{P}_{n} \rightarrow X$ for some non-negative integer $n$; we define $s(\alpha)=\alpha(0)$ and $t(\alpha)=\alpha(n)$. If $a$ is an arc in $X$ such that $t(\alpha)=s(a)$, then we define $\alpha a: \mathbf{P}_{n+1} \rightarrow X$, the concatenation of $\alpha$ and $a$.

Let us introduce some useful shorthand for arcs in a graph $X$. For any node $x$ in a graph $X$, let $X(x, *)$ denote the set of those $\operatorname{arcs}$ in $X$ which have source $x$, and let $X(*, x)$ denote the set of $\operatorname{arcs}$ with target $x$. Note that a graph morphism $f: X \rightarrow Y$ induces a function $f: X(x, *) \rightarrow Y(f(x), *)$, etc.

Here is our first class of graph morphisms.

Definition 3.1. A graph morphism $f: X \rightarrow Y$ is Surjecting if

$$
f: X(x, *) \rightarrow Y(f(x), *)
$$

is a surjective function for all $x \in X_{0}$.

A discrete graph is one with no arcs. We say that a node $x$ is a root of the graph $X$ if $X(*, x)$ is empty. Let $R(X)$ denote the set of roots in $X$, viewed as a discrete subgraph of $X$. A rooted tree is a graph $T$ with one root $r$ such that, for each node $x$ in $T$, there is a unique (directed) path in $T$ from $r$ to $x$. For example, the path graph $\mathbf{P}_{n}$ is a rooted tree; and so is the infinite path $\mathbf{P}_{\infty}$ whose nodes are the set of nonnegative integers and whose arcs are the set of ordered-pairs $(i-1, i)$ of non-negative integers, with $s((i-1, i))=i-1$ and $t((i-1, i))=i$.

For any node $x$ in a graph $X$, we can define a rooted tree $T_{x} X$, the tree of paths in $X$ leaving $x$. The nodes in $T_{x} X$ are the finite paths in $X$ with source $x$ (note that $x$ is considered as a path of length 0 in $X)$; the $\operatorname{arcs}$ in $T_{x} X$ are the triples $(\alpha, a, \alpha a)$ 
where $\alpha a$ is the concatenation of path $\alpha$ and $\operatorname{arc} a$ in $X$; and $s(\alpha, a, \alpha a)=\alpha$ and $t(\alpha, a, \alpha a)=\alpha a$. There are natural graph morphisms $T_{x} X \rightarrow X$ given by $\alpha \mapsto t(\alpha)$ and $(\alpha, a, \alpha a) \mapsto a$.

A rooted forest $F$ is a coproduct of rooted trees. If $F$ is a forest with roots $R(F)$, then we may form a new graph $X_{F}$ as the pushout of the graph morphisms $r: R(F) \rightarrow F$ and $f: R(F) \rightarrow X$; we say that $X_{F}$ is formed by attaching the forest $F$ to $X$ along $R$. For instance, if $r$ is a root in a tree $T$ and $x$ is any node in a graph $X$, then $X_{T}$ is formed by attaching the tree $T$ to $X$ at the node $x$ (along the graph morphisms $r: \mathbf{D} \rightarrow T$ and $x: \mathbf{D} \rightarrow X$ ).

Definition 3.2. A graph morphism $f: X \rightarrow Y$ is a Whiskering if $Y$ is formed by attaching some rooted forest to $X$.

For example, the graph morphism $\mathbf{s}: \mathbf{D} \rightarrow \mathbf{A}$ is a Whiskering, where $\mathbf{s}$ exhibits the "dot" graph D as the source subgraph of the "arrow" graph A. Also, every isomorphism is a Whiskering, and $R(F) \rightarrow F$ is a Whiskering if $F$ is a rooted forest.

Our goal in this section is to demonstrate some remarkable factorization properties of Surjectings and Whiskerings. Here is the conceptual background.

Definition 3.3. Let $\ell: X \rightarrow Y$ and $r: A \rightarrow B$ be morphisms in a category $\mathcal{S}$. We say that $\ell \dagger r$ when, for all $f$ and $g$, if

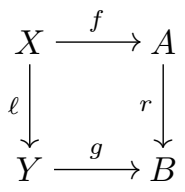

commutes, then

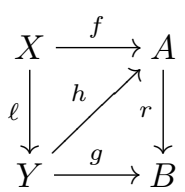

commutes for some $h$. We say that $h$ is a filler for the commutative diagram. We may also say that $h$ lifts $g$ along $r$, or that $h$ drops ("extends") $f$ along $\ell$. Given two classes $\mathcal{L}$ and $\mathcal{R}$ of morphisms, we say $\mathcal{L} \dagger \mathcal{R}$ when we have $\ell \dagger r$ for every $\ell \in \mathcal{L}$ and every $r \in \mathcal{R}$. Given a class $\mathcal{F}$ of morphisms we may define

$$
\mathcal{F}^{\dagger}=\{r: f \dagger r, \forall f \in \mathcal{F}\} \quad \text { and } \quad \dagger \mathcal{F}=\{\ell: \ell \dagger f, \forall f \in \mathcal{F}\} .
$$

Definition 3.4. A weak factorization system in $\mathcal{S}$ is given by two classes $\mathcal{L}$ and $\mathcal{R}$ such that $\mathcal{L}^{\dagger}=\mathcal{R}$ and $\mathcal{L}={ }^{\dagger} \mathcal{R}$ and such that, for any morphism $c$ in $\mathcal{S}$, there exist $\ell \in \mathcal{L}$ and $r \in \mathcal{R}$ with $c=r \circ \ell$.

The notion of a weak factorization system has become a part of homotopical algebra; see Section 4.

The following three propositions combine to show that Surjectings and Whiskerings give a weak factorization system in Gph. Our Proposition 3.5 was inspired by an argument in Enochs and Herzog [4]. 
Proposition 3.5. Any graph morphism $f: X \rightarrow Y$ factors as a Whiskering followed by a Surjecting.

Proof. Recall that for each node $y$ in $Y$ we have the tree $T_{y} Y$ of paths in $Y$ leaving $y$. From $f$ we construct the rooted forest $F=\sum_{x \in X_{0}} T_{f(x)} Y$, with roots $R=X_{0}$ considered as a discrete subgraph of $X$. The pushout of $R \rightarrow F$ along the subgraph inclusion $R \rightarrow X$ defines a Whiskering $w: X \rightarrow X_{F}$. We have $g: F \rightarrow Y$ as a coproduct of the morphisms $T_{f(x)} Y \rightarrow Y$, and since $f: X \rightarrow Y$ and $g: F \rightarrow Y$ agree on $R$, they determine a unique graph morphism $p: X_{F} \rightarrow Y$. Note that $f=p \circ w$.

Let us show that $p$ is a Surjecting. For any node $z$ in $X_{F}$ we must show that $p: X_{F}(z, *) \rightarrow Y(p(z), *)$ is surjective. But $z$ is either a node $x$ or a path in $Y$ with source $f(x)$, for some $x \in X_{0}$.

In the first case, we have $p: X_{F}(x, *) \rightarrow Y(f(x), *)$ with

$$
X_{F}(x, *)=X(x, *) \cup T_{f(x)} Y(f(x), *),
$$

and $T_{f(x)} Y(f(x), *) \rightarrow Y(f(x), *)$ is a bijection.

In the second case, we have $p: X_{F}(\alpha, *) \rightarrow Y(p(\alpha), *)$ with

$$
X_{F}(\alpha, *)=T_{f(x)} Y(\alpha, *)=Y(t(\alpha), *)=Y(p(\alpha), *) .
$$

In either case, $p: X_{F}(z, *) \rightarrow Y(p(z), *)$ is surjective.

Proposition 3.6. Whiskering $\dagger$ Surjecting.

Proof. Let $f: Z \rightarrow Y$ be Surjecting. First we show lifting of rooted trees. More precisely, if $T$ is a rooted tree with root $x$ and we have the following commutative diagram

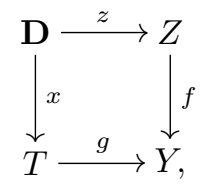

then there is a filler $h: T \rightarrow Z$. This follows by induction on the length of path from the root to nodes of $T$, as follows. Suppose that we have extended $h$ to paths of length $n$ and let $\alpha a$ be a path of length $n+1$ in $T$. Let $x^{\prime}=t(\alpha)$. Then $f\left(h\left(x^{\prime}\right)\right)=g\left(x^{\prime}\right)$, $g(a) \in Y\left(g\left(x^{\prime}\right), *\right)$ and $f: Z\left(h\left(x^{\prime}\right), *\right) \rightarrow Y\left(g\left(x^{\prime}\right), *\right)$ is a surjective function, so there exists an arc $a^{\prime} \in Z\left(h\left(x^{\prime}\right), *\right)$ so that $f\left(a^{\prime}\right)=g(a)$. We extend $h$ to $\alpha a$ by $h(a)=a^{\prime}$.

More generally, consider any commutative diagram

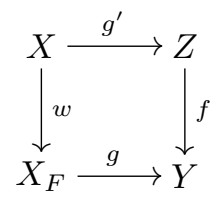

with $w$ a Whiskering given by

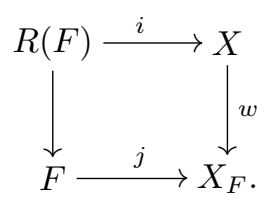


We want to define a filler $h: X_{F} \rightarrow Z$, by extending $g^{\prime}$ along every tree $T$ in $F$. This is possible since the square is commutative and $f$ is Surjecting.

Proposition 3.7. (Whiskering, Surjecting) is a weak factorization system.

Proof. By the preceding proposition we have

$$
\text { Whiskering } \subseteq{ }^{\dagger} \text { Surjecting and Surjecting } \subseteq \text { Whiskering }{ }^{\dagger} \text {. }
$$

If $f: X \rightarrow Y$ is not in Surjecting, then there exists some $x \in X_{0}$ and some $a \in$ $Y(f(x), *)$ which is not in the image of $X(x, *) \rightarrow Y(f(x), *)$. Consider the Whiskering $\mathbf{s}: \mathbf{D} \rightarrow \mathbf{A}$ and the commutative diagram

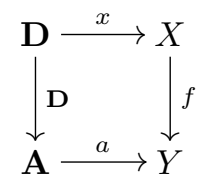

for which there is no filler. This shows that $f \notin$ Surjecting implies $f \notin$ Whiskering $^{\dagger}$. It follows that Whiskering ${ }^{\dagger}=$ Surjecting. We will show that $f \notin$ Whiskering implies $f \notin \dagger$ Surjecting, by factoring. Suppose that $f: X \rightarrow Y$ with $f \notin$ Whiskering; then $f=p \circ w$ with some Whiskering $w: X \rightarrow X_{F}$ and some Surjecting $p: X_{F} \rightarrow Y$. Consider the commutative diagram

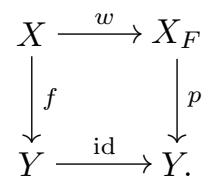

If this had a filler $h: Y \rightarrow X_{F}$, then we would have $p \circ h=\mathrm{id}$. This would exhibit $f$ as a "morphism retract" of $w$ (a retract in the morphism category). But we show in the next lemma that this would give us the desired contradiction, finishing our proof.

Lemma 3.8. Whiskerings are stable with respect to morphism retract.

Proof. First we show that any retract of a rooted tree is a rooted tree. If $T$ is a rooted tree with root $x_{0}$ and we have the following commutative diagram with $r \circ s=\mathrm{id}_{T^{\prime}}$

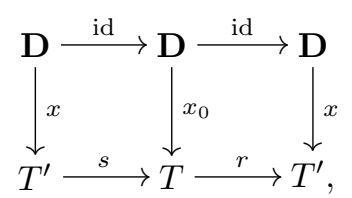

then $T^{\prime}$ is a rooted tree with root $x$. This is clear since, for any node $x^{\prime}$ in $T^{\prime}$, the unique path $\alpha$ from $x_{0}$ to $s\left(x^{\prime}\right)$ gives $r \circ \alpha$ a path from $x$ to $r\left(s\left(x^{\prime}\right)\right)=x^{\prime}$, and there can be no other path in $T^{\prime}$ from $x$ to $x^{\prime}$. 
More generally, consider any commutative diagram

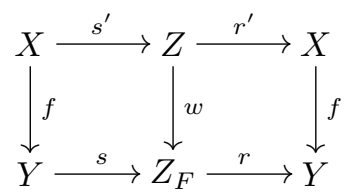

such that $w$ is a Whiskering, $r \circ s=\operatorname{id}_{Y}$ and $r^{\prime} \circ s^{\prime}=\operatorname{id}_{X}$. The fact that $r \circ s=\operatorname{id}_{Y}$ implies that $s$ is injective on nodes and arcs. Also $w$ is injective on nodes and arcs since it is a Whiskering. This implies that $f$ is injective on nodes and arcs, since the first square is commutative.

We will describe a rooted forest $F^{\prime}$ with roots $R^{\prime}$ a discrete subgraph of $X$, such that $Y=X_{F^{\prime}}$.

The Whiskering $w$ is given by a rooted forest $F$ whose roots $R$ form a discrete subgraph of $Z$. Let $R^{\prime}=R \cap X$ as subgraphs of $Z$. Then $R^{\prime}$ is a discrete subgraph of $X$. For each $x \in R^{\prime}$, consider the tree $T$ in the forest $F$ with root $x_{0}=s(x)$. Then $r(T)$ is a retract of $T$, so $r(T)$ is a tree with root $x=r\left(x_{0}\right)$. Let $F^{\prime}=\sum_{x \in R^{\prime}} r(T)$. Then $Y=X_{F^{\prime}}$, and we are done.

\section{A Quillen model structure on the category of graphs}

Suppose that $\mathcal{S}$ is a category with finite limits and finite colimits. Then Quillen's notion [14] of "model category" can be expressed via the following axioms (which we learned from Section 7 of Joyal and Tierney $[\mathbf{8}]$ ).

Definition 4.1. A model structure on $\mathcal{S}$ is a triple $(\mathcal{C}, \mathcal{W}, \mathcal{F})$ of classes of morphisms in $\mathcal{S}$ that satisfies:

(1) "three for two": if two of the three morphisms $a, b, a \circ b$ belong to $\mathcal{W}$, then so does the third;

(2) the pair $(\underline{\mathcal{C}}, \mathcal{F})$ is a weak factorization system (where $\underline{\mathcal{C}}=\mathcal{C} \cap \mathcal{W}$ );

(3) the pair $(\mathcal{C}, \underline{\mathcal{F}})$ is a weak factorization system (where $\underline{\mathcal{F}}=\mathcal{W} \cap \mathcal{F}$ ).

The morphisms in $\mathcal{W}$ are called weak equivalences. The morphisms in $\mathcal{C}$ are called cofibrations, and the morphisms in $\underline{\mathcal{C}}$ are called acyclic cofibrations. The morphisms in $\mathcal{F}$ are called fibrations, and the morphisms in $\underline{\mathcal{F}}$ are called acyclic fibrations.

Note that, according to Hovey [6, p. 28], "It tends to be quite difficult to prove that a category admits a model structure. The axioms are always hard to check."

Recall from Section 3 that the path graph $\mathbf{P}_{n}$ has nodes $\{0, \ldots, n\}$. For $n \geqslant 0$, the cycle graph $\mathbf{C}_{n}$ is the graph produced by identifying the nodes 0 and $n$ of $\mathbf{P}_{n}$. We have $\mathbf{C}_{0}=\mathbf{P}_{0}=\mathbf{D}$, the graph with one node and no arcs; and $\mathbf{C}_{1}$ is the graph with one node, and one arc with source equal to target. Let $C_{n}(X)$ denote the set of graph morphisms from $\mathbf{C}_{n}$ to $X$; we may call this the set of $n$-cycles in $X$.

Definition 4.2. A graph morphism $f: X \rightarrow Y$ is Acyclic when

$$
C_{n}(f): C_{n}(X) \rightarrow C_{n}(Y)
$$

is bijective for all $n>0$. 
Here we exclude $n=0$, since we do not want to require that $f_{0}: X_{0} \rightarrow Y_{0}$ is a bijection.

The Acyclics contain the Whiskerings, and many other useful graph morphisms.

Now we are ready to define the morphism classes $\mathcal{W}$ and $\mathcal{C}$ and $\mathcal{F}$ for our Quillen model structure on Gph.

Definition 4.3. Let $\mathcal{W}$ be the Acyclics. Let $\mathcal{F}$ be the Surjectings, so that $\underline{\mathcal{F}}=\mathcal{W} \cap \mathcal{F}$ must be the Acyclic Surjectings. Let $\mathcal{C}$ be ${ }^{\dagger} \underline{\mathcal{F}}$.

In the next few propositions we show directly that this does indeed define a model structure on Gph. We use the following facts, which are easy to verify directly from the definition $\mathcal{C}={ }^{\dagger} \underline{\mathcal{F}}$ :

(1) Every composition of graph morphisms in $\mathcal{C}$ is in $\mathcal{C}$.

(2) Every Whiskering is in $\mathcal{C}$.

(3) For any set $I$ and $n>0$, if $A_{i} \rightarrow B_{i}$ is in $\mathcal{C}$ for all $i \in I$, then $\sum_{i \in I} A_{i} \rightarrow \sum_{i \in I} B_{i}$ is in $\mathcal{C}$.

(4) For any set $I$ and $n>0$, the graph morphism i: $0 \rightarrow I \times \mathbf{C}_{n}$ is in $\mathcal{C}$.

(5) For any set $I$, the graph morphism $\mathbf{j}: I \times \mathbf{C}_{n} \rightarrow \mathbf{C}_{n}$ is in $\mathcal{C}$.

In (4) and (5) we view the set $I$ as a discrete graph and we view the graph $I \times \mathbf{C}_{n}$ as a sum of copies of $\mathbf{C}_{n}$. Let $\mathbf{i}_{n}$ denote $\mathbf{i}: 0 \rightarrow \mathbf{C}_{n}$ and let $\mathbf{j}_{n}$ denote $\mathbf{j}: \mathbf{C}_{n}+\mathbf{C}_{n} \rightarrow \mathbf{C}_{n}$.

Proposition 4.4. The Acyclics satisfy the "three for two" property.

Proof. This is easy, since Acyclics are defined functorially. Consider $h=f \circ g$. Then $C_{n}(h)=C_{n}(f) \circ C_{n}(g)$ for all $n$. But the class of bijective functions in the category of sets satisfies the "three for two" property.

Proposition 4.5. $(\mathcal{C}, \underline{\mathcal{F}})$ is a weak factorization system in $\mathrm{Gph}$.

Proof. We have already shown in Section 3 that (Whiskering, Surjecting) is a weak factorization system in Gph. Let us show that the class of Whiskerings is equal to $\underline{\mathcal{C}}=\mathcal{C} \cap \mathcal{W}$. Every Whiskering is in $\underline{\mathcal{C}}$. Suppose $f \in \underline{\mathcal{C}}$. By Proposition 3.5 we have $f=p \circ w$ with $p \in \mathcal{F}$, so the diagram

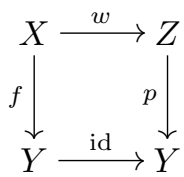

has a filler $h: Y \rightarrow Z$ with $h \circ f=w$ and $p \circ h=\operatorname{id}_{Y}$. It follows that

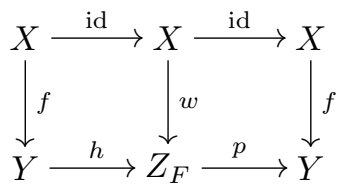

commutes, making $f$ a retract of the Whiskering $w$. Thus $f$ is a Whiskering, by Lemma 3.8. 
Proposition 4.6. Every graph morphism $g$ factors as $g=f \circ c$ with

$$
c \in \mathcal{C} \text { and } f \in \underline{\mathcal{F}} \text {. }
$$

Proof. Given any graph morphism $g: X \rightarrow Y$, we factor $g$ in three steps.

First, we let $C$ be the disjoint union of a copy of $\mathbf{C}_{n}$ for each element of $C_{n}(Y)$ which is not in the image of $C_{n}(g): C_{n}(X) \rightarrow C_{n}(Y)$. Let $h: C \rightarrow Y$ be the graph morphism which sends each summand cycle of $C$ to its image in $Y$. Let $X^{\prime}=X+C$, let $g^{\prime}: X \rightarrow X^{\prime}$ denote the inclusion $X \rightarrow X+C$, and let $f^{\prime}: X^{\prime} \rightarrow Y$ denote the graph morphism $X+C \rightarrow Y$ determined by $g: X \rightarrow Y$ and $h: C \rightarrow Y$. Then $g=f^{\prime} \circ g^{\prime}$, and $g^{\prime} \in \mathcal{C}$, and $C_{n}\left(f^{\prime}\right): C_{n}\left(X^{\prime}\right) \rightarrow C_{n}(Y)$ is surjective for all $n>0$.

Next, we let $J=\left\{(c, n): c \in C_{n}(Y)\right\}$, with $j: \sum_{J} I_{c} \times \mathbf{C}_{n} \rightarrow \sum_{J} \mathbf{C}_{n}$, where $I_{c}$ is the preimage of $c$ for the function $C_{n}\left(f^{\prime}\right): C_{n}\left(X^{\prime}\right) \rightarrow C_{n}(Y)$. Also let $k: \sum_{J} I_{c} \times$ $\mathbf{C}_{n} \rightarrow X^{\prime}$ be the graph morphism which sends each summand cycle to the corresponding cycle in $X^{\prime}$, and let $\ell: \sum_{J} \mathbf{C}_{n} \rightarrow Y$ be the graph morphism which sends each summand cycle to the corresponding cycle in $Y$. Let $g^{\prime \prime}: X^{\prime} \rightarrow X^{\prime \prime}$ denote the pushout of $j$ along $k$. Let $f^{\prime \prime}: X^{\prime \prime} \rightarrow Y$ be the pushout graph morphism induced by $\ell$ and $f^{\prime}$. Then $g^{\prime \prime} \in \mathcal{C}$ and $f^{\prime}=f^{\prime \prime} \circ g^{\prime \prime}$, and $C_{n}\left(f^{\prime \prime}\right): C_{n}\left(X^{\prime \prime}\right) \rightarrow C_{n}(Y)$ is bijective for all $n>0$, so that $f^{\prime \prime} \in \mathcal{W}$.

Finally, we factor $f^{\prime \prime}=f^{\prime \prime \prime} \circ g^{\prime \prime \prime}$ with Whiskering $g^{\prime \prime \prime}: X^{\prime \prime} \rightarrow X^{\prime \prime \prime}$ and Surjecting $f^{\prime \prime \prime}: X^{\prime \prime \prime} \rightarrow Y$, as in Section 3. Then $g^{\prime \prime \prime} \in \mathcal{C}$ and $f^{\prime \prime \prime} \in \mathcal{W} \cap \mathcal{F}$.

Thus, $g=c \circ f$ with $c=g^{\prime \prime \prime} \circ g^{\prime \prime} \circ g^{\prime}$ in $\mathcal{C}$, and $f=f^{\prime \prime \prime}$ in $\underline{\mathcal{F}}$.

Proposition 4.7. $(\mathcal{C}, \underline{\mathcal{F}})$ is a weak factorization system in $\mathrm{Gph}$.

Proof. We have $\mathcal{C}={ }^{\dagger} \underline{\mathcal{F}}$, by definition. This shows also that $\underline{\mathcal{F}} \subseteq \mathcal{C}^{\dagger}$. It remains only to show that $\mathcal{C}^{\dagger} \subseteq \underline{\mathcal{F}}$. But this is easy. Consider the Whiskering $\mathbf{s}: \mathbf{D} \rightarrow \mathbf{A}$ from Section 3 , and the graph morphisms $\mathbf{i}_{n}: 0 \rightarrow \mathbf{C}_{n}, \mathbf{j}_{n}: \mathbf{C}_{n}+\mathbf{C}_{n} \rightarrow \mathbf{C}_{n}$ as in (4) and (5) above. These are all in $\mathcal{C}$, since each can be lifted against any graph morphism in $\underline{\mathcal{F}}$. But if $g \notin \underline{\mathcal{F}}$, then we can show failure of lifting for either $\mathbf{s}$ or some $\mathbf{i}_{n}$ or $\mathbf{j}_{n}$.

Corollary 4.8. Our morphism classes $\mathcal{W}$ and $\mathcal{C}$ and $\mathcal{F}$ provide a Quillen model structure for the category Gph.

The above proofs show how the graph morphisms $\mathbf{s}: \mathbf{D} \rightarrow \mathbf{A}$, together with $\mathbf{i}_{n}: 0 \rightarrow$ $\mathbf{C}_{n}$ and $\mathbf{j}_{n}: \mathbf{C}_{n}+\mathbf{C}_{n} \rightarrow \mathbf{C}_{n}$, for $n>0$, generate our class $\mathcal{C}$ of cofibrations. This situation is a special case of a general principle in presheaf categories; see Proposition 7.5 in Joyal and Tierney [8], for instance.

\section{Zeta series and almost isospectral graphs}

Ihara zeta functions of graphs are usually discussed in a setting of "unoriented" or "symmetric" graphs; see Kotani and Sunada [9], for instance. We need a version suitable for directed graphs (in this section we may refer to objects of our category Gph as directed graphs, for emphasis). There is a nice treatment of zeta series of finite directed graphs in Section 2 of Kotani and Sunada [9]; we will follow them here, but with our own terminology. 
Definition 5.1. A finite graph is one with finitely many nodes and arcs. The zeta series of a finite directed graph $X$ is the formal power series

$$
Z(u)=\exp \left(\sum_{m=1}^{\infty} c_{m} \frac{u^{m}}{m}\right)
$$

where $c_{m}=\left|C_{m}(X)\right|$ for $m>0$.

See the appendix for some motivation for this definition, including how it relates to an Euler product expansion in terms of "primes".

Example 5.2. If $X$ is the graph with one node and $n$ arcs, then $c_{m}=n^{m}$ and

$$
\sum_{m=1}^{\infty} c_{m} \frac{u^{m}}{m}=\sum_{m=1}^{\infty} \frac{n^{m} u^{m}}{m}=-\log (1-n u) \quad \text { so that } \quad Z(u)=\frac{1}{1-n u} .
$$

Definition 5.3. Let $X$ be a finite graph. Let $\mathrm{R} X_{0}$ denote the real vector space with basis the nodes of $X$. The adjacency operator $A$ for $X$ is the linear transformation $A: \mathrm{R} X_{0} \rightarrow \mathrm{R} X_{0}$ determined by

$$
A(x)=\sum_{a \in X(x, *)} t(a)
$$

for $x \in X_{0}$. The characteristic polynomial of $X$ is defined as $a(x)=\operatorname{det}(x I-A)$, the characteristic polynomial of the adjacency operator $A$ for $X$. If $X$ has $n$ nodes, then $a(x)$ is a monic polynomial of degree $n$, and the reversed characteristic polynomial of $X$ is defined to be $u^{n} a\left(u^{-1}\right)=\operatorname{det}(I-u A)$.

Note that the reversed characteristic polynomial of a finite graph $X$ has constant term 1 , and is thus a unit in the ring of formal power series with integer coefficients.

If we totally order the nodes of $X$, then the adjacency operator is represented by the square matrix $A$ with entry $A_{j, i}$ equal to the number of arcs in $X$ from the $i^{\text {th }}$ node to the $j^{\text {th }}$ node. It follows that $c_{m}=\left|C_{m}(X)\right|$ is the trace of the matrix $A^{m}$.

Proposition 5.4. If $X$ is a finite graph with $n$ nodes and $Z(u)$ is the zeta series of $X$, then

$$
Z(u)=\operatorname{det}(I-u A)^{-1}=\frac{1}{u^{n} a\left(u^{-1}\right)} .
$$

Proof. Let $A$ be any endomorphism of an $n$-dimensional real vector space $V$. We have $\operatorname{det}(I-u A)=u^{n} \operatorname{det}\left(u^{-1} I-A\right)$, which proves the second equality. The first equality follows from

$$
\exp \left(\sum_{m=1}^{\infty} \operatorname{Trace}\left(A^{m}\right) \frac{u^{m}}{m}\right)=\operatorname{det}(1-u A)^{-1} .
$$

One can check this linear algebra identity by induction on the dimension of $V$, since both sides are multiplicative for short exact sequences of vector spaces endowed with endomorphisms. Or, when $V$ has a basis of eigenvectors for $A$ with eigenvalues $\lambda_{1}, \ldots, \lambda_{n}$, the identity follows from $-\log (1-x)=\sum_{k} \frac{x^{k}}{k}$ and

$$
\exp \left(\sum_{m=1}^{\infty} \sum_{i=1}^{n} \lambda_{i}^{m} \frac{u^{m}}{m}\right)=\prod_{i=1}^{n} \exp \left(-\log \left(1-\lambda_{i} u\right)=\prod_{i=1}^{n} \frac{1}{1-\lambda_{i} u}=\operatorname{det}(I-u A)^{-1}\right.
$$


Example 5.5 (continued). If $X$ is the graph with one node and $n \operatorname{arcs,~then~} a(x)=$ $x-n$ and $u^{1} a\left(u^{-1}\right)=1-n u$, which agrees with $Z(u)=\frac{1}{1-n u}$.

Proposition 5.6. If $X$ and $Y$ are finite graphs and $f: X \rightarrow Y$ is an acyclic morphism, then $Z_{X}=Z_{Y}$.

Proof. This is clear from the definition, since $\left|C_{m}(X)\right|=\left|C_{m}(Y)\right|$ for all $m>0$.

Definition 5.7. The eigenvalues of the adjacency operator for $X$ may be called the spectrum of $X$ (even though the adjacency operator is not necessarily a diagonalizable operator). We say that two finite graphs $X$ and $Y$ are isospectral if they have the same characteristic polynomial. We say that $X$ and $Y$ are almost isospectral if they have the same reversed characteristic polynomial.

Loosely speaking, $X$ and $Y$ are almost isospectral if and only if they have the same non-zero eigenvalues.

Corollary 5.8. If $X$ and $Y$ are finite graphs with $Z_{X}=Z_{Y}$, then $X$ and $Y$ are almost isospectral.

Proof. This follows immediately from the preceding two propositions.

\section{Appendix A. An appendix on the zeta series and its Euler product expansion}

Here is a little history, based on Thomas [16], of how our zeta series for finite directed graphs relate to the famous zeta functions from number theory.

\section{The zeta function of Euler and Riemann.}

Let $p$ range over the prime numbers. Then

$$
\zeta(s)=\sum_{n} \frac{1}{n^{s}}=\prod_{p}\left(1-\frac{1}{p^{s}}\right)^{-1} .
$$

\section{Dedekind's zeta function for algebraic number fields.}

Let $A$ be the ring of integers in an algebraic number field $K$ (so $K$ is a finite extension over the field $\mathrm{Q}$ of rational numbers). Let $N(I)=|A / I|$ for any non-zero ideal in $A$. Then

$$
\zeta(s)=\sum_{I} \frac{1}{N(I)^{s}}=\prod_{P}\left(1-N(P)^{-s}\right)^{-1},
$$

where $I$ ranges over the non-zero principal ideals in $A$ and $P$ ranges over the prime ideals in $A$.

\section{A zeta function for algebraic function fields.}

Let $A$ be the ring of integers in an algebraic function field (so $K$ is a finite extension over the field $\mathrm{F}_{q}(x)$ of rational functions with coefficients in the field $\mathrm{F}_{q}$ with $q$ elements). But here $N(I)=|A / I|=q^{\nu(I)}$ for any non-zero ideal in $A$, where $\nu(I)$ is 
the dimension of $A / I$ as a finite-dimensional vector space over $F_{q}$, so that

$$
\zeta(s)=\left.Z(u)\right|_{u=q^{-s}} \quad \text { for } \quad Z(u)=\prod_{P}\left(1-u^{\nu(P)}\right)^{-1} .
$$

The zeta function for a projective variety over a finite field from Weil [18] is a completed version of this.

The form of zeta series that we use in Section 5 seems ultimately based on the following observation. If $A$ is an endomorphism of a finite-dimensional vector space over a field of characteristic zero, then the knowledge of the trace of $A^{n}$ for all $n$ is equivalent to the knowledge of the reversed characteristic polynomial of $A$, as we see from the identity

$$
\operatorname{det}(1-u A)^{-1}=\exp \left(\sum_{n=1}^{\infty} \operatorname{Trace}\left(A^{n}\right) \frac{u^{n}}{n}\right)
$$

used in the proof of Proposition 5.4.

If $A$ is a matrix expressing a function $\tau: S \rightarrow S$ with $S$ finite, then the trace of $A^{n}$ counts the number of fixed points of $\tau^{n}$. This is one of the ideas behind the Lefschetz fixed point theorem, the Weil zeta function used in the Weil conjectures, and other dynamical zeta functions such as the Selberg zeta function in Riemannian geometry and the Ihara zeta function (see Ruelle [15] for instance).

In our setting of graphs, we merely use that if $A$ is the adjacency matrix of a finite graph $X$, then the trace of $A^{n}$ counts the number of cycles of length $n$ in $X$. This leads to the following Euler product expansion, analogous to the one for algebraic function fields.

The cycle graph $\mathbf{C}_{n}$ has nodes $i$ for $0 \leqslant i<n$. If $m$ divides $n$ then we have a graph morphism $\pi: \mathbf{C}_{n} \rightarrow \mathbf{C}_{m}$ given by sending node $i$ to node $i \bmod m$.

Definition A.1. A cycle $c: \mathbf{C}_{k m} \rightarrow X$ is a $k$-multiple if $c=c^{\prime} \circ \pi$ for some cycle $c^{\prime}: \mathbf{C}_{m} \rightarrow X$. A prime cycle of length $n$ in $X$ is a cycle $c: \mathbf{C}_{n} \rightarrow X$ which is not a $k$-multiple for any $k>1$. Let us say that two cycles $c, c^{\prime}: \mathbf{C}_{n} \rightarrow X$ are shift equivalent if $c^{\prime}=c \circ \tau^{i}$ for some $i$, where $\tau^{i}: \mathbf{C}_{n} \rightarrow \mathbf{C}_{n}$ is the shift morphism sending node $j$ to node $j+i \bmod n$. Let us say that a prime $P$ in $X$ is an equivalence class of prime cycles in $X$, and that $\nu(P)$ is the length of the prime $P$.

This makes sense, since shift equivalence is an equivalence relation on $C_{n}(X)$, and on prime cycles of length $n$ in $X$.

Proposition A.2. The Euler product expansion for the zeta function of a finite graph is given by

$$
Z(u)=\prod_{P}\left(1-u^{\nu(P)}\right)^{-1},
$$

where $P$ ranges over all primes in $X$ and $\nu(P)$ is the length of $P$. 
Proof. Let $\bar{c}_{k}$ be the number of primes $P$ of length $k$. Then $c_{m}=\sum_{\{k: k \mid m\}} k \bar{c}_{k}$ and we have

$$
\begin{aligned}
\log \prod_{P}\left(1-u^{\nu(P)}\right)^{-1} & =\sum_{P} \sum_{k=1}^{\infty} \frac{u^{k|p|}}{k}=\sum_{k=1}^{\infty} \sum_{\ell=1}^{\infty} \sum_{\nu(P)=\ell} \frac{u^{k \ell}}{k} \\
& =\sum_{k=1}^{\infty} \sum_{\ell=1}^{\infty} \bar{c}_{\ell} \frac{u^{k \ell}}{k}=\sum_{m=1}^{\infty} \frac{1}{m} \sum_{\ell \mid m} \ell \bar{c}_{\ell} u^{m}=\sum_{m=1}^{\infty} c_{m} \frac{u^{m}}{m}
\end{aligned}
$$

which is $\log Z(u)$. Here equality one and equality four follow from

$$
-\log \left(1-u^{v}\right)=\sum_{k} \frac{u^{k v}}{k} \quad \text { and } \quad m=k \ell \Longleftrightarrow \frac{\ell}{m}=\frac{1}{k} .
$$

Example A.3 (Example 5.2 (continued)). For $X$ the graph with one node and two arcs, we must have

$$
\frac{1}{1-2 u}=(1-u)^{-2}\left(1-u^{2}\right)^{-1}\left(1-u^{3}\right)^{-2} \cdots=\prod\left(1-u^{k}\right)^{-\bar{c}_{k}}
$$

This is related to the cyclotomic (necklace) identity; see Dress and Siebeneicher [2], for instance.

\section{References}

[1] D-C. Cisinski, Théories homotopiques dans les topos, J. Pure Appl. Algebra 174 (2002), no. 1, 43-82.

[2] A.W.M. Dress and C. Siebeneicher, The Burnside ring of the infinite cyclic group and its relations to the necklace algebra, $\lambda$-rings, and the universal ring of witt vectors, Adv. Math. 78 (1989), 1-41.

[3] W.G. Dwyer and J. Spalinski, Homotopy theories and model categories, in Handbook of algebraic topology (I.M. James, ed.), 73-126, Elsevier B V, Amsterdam, 1995.

[4] E.E. Enochs and I. Herzog, A homotopy of quiver morphisms with applications to representations, Canadian J. Math. 51 (1999), no. 2, 294-308.

[5] L. Fajstrup and J. Rosický, A convenient category for directed homotopy, in Theory and applications of categories 21 (2008), no. 1, 7-20.

[6] M. Hovey, Model categories, Math. Surveys and Monographs 63, Amer. Math. Soc., Providence, RI, 1999.

[7] A. Joyal and M. Tierney, Strong stacks and classifying spaces, in Category theory (Como, 1990), Lecture Notes in Math. 1488, 213-236, Springer-Verlag, New York, 1991.

[8] A. Joyal and M. Tierney, Quasi-categories vs Segal spaces, in Categories in algebra, geometry and mathematical physics, Contemp. Math. 431, 277-326, Amer. Math. Soc., Providence, RI, 2007.

[9] M. Kotani and T. Sunada, Zeta functions of finite graphs, J. Math. Sci. Univ. Tokyo 7 (2000), no. 1, 7-25. 
[10] F.W. Lawvere, Qualitative distinctions between some toposes of generalized graphs, in Categories in computer science and logic (Boulder 1987), Contemp. Math. 92, 261-299, Amer. Math. Soc., Providence, RI, 1989.

[11] F.W. Lawvere and S.H. Schanuel, Conceptual mathematics: a first introduction to categories, Cambridge University Press, Cambridge, 1997.

[12] S. Mac Lane and I. Moerdijk, Sheaves in geometry and logic: a first introduction to topos theory, Universitext, Springer-Verlag, New York (1994).

[13] F. Morel and V. Voevodsky, $A^{1}$-homotopy of schemes, Inst. Hautes Études Sci. Publ. Math. 90 (1999), 45-143.

[14] D.G. Quillen, Homotopical algebra, Lecture Notes in Math. 43, SpringerVerlag, New York, 1967.

[15] D. Ruelle, Dynamical zeta functions and transfer operators, Notices Amer. Math. Soc. 49 (2002), no. 8, 887-895.

[16] A.D. Thomas, Zeta-functions: an introduction to algebraic geometry, Pitman, London, 1977.

[17] R.W. Thomason, Cat as a closed model category, Cahiers Topologie Géom. Différentielle 21 (1980), no. 3, 305-324.

[18] A. Weil, Number of solutions of equations in finite fields, Bull. Amer. Math. Soc. 55 (1949), 497-508.

Terrence Bisson bisson@canisius.edu

Department of Mathematics and Statistics, Canisius College, 2001 Main St., Buffalo, NY 14208, USA

Aristide Tsemo tsemo58@yahoo.ca

Mathematics, College Boreal, 351 Carlaw Ave., Toronto, Ontario M4K 3M2, Canada Or: 102 Goodwood Park, Apt. 614, Toronto, Ontario M4C 2G8, Canada 